\title{
A matched comparison of the patient-reported outcome measures of cemented and cementless total knee replacements, based on the National Joint Registry of England, Wales, Northern Ireland, and Isle of Man and England's National PROM collection programme
}

\author{
Hasan R MOHAMMAD ${ }^{1}$, Andrew JUDGE ${ }^{2}$, and David W MURRAY ${ }^{1}$
}

\author{
${ }^{1}$ Nuffield Department of Orthopaedics, Rheumatology and Musculoskeletal Sciences University of Oxford, Nuffield Orthopaedic Centre, Oxford; \\ ${ }^{2}$ Musculoskeletal Research Unit, Bristol Medical School, University of Bristol, UK \\ Correspondence: hasanmohammad@doctors.org.uk \\ Submitted 2021-08-18. Accepted 2021-10-28
}

Background and purpose - Total knee replacement (TKR) can be implanted with or without bone cement. It is currently unknown how the functional outcomes compare. Therefore, we compared the patient-reported outcome measures (PROMS) of both fixation methods.

Patients and methods - We performed a propensitymatched comparison of 14,404 TKRs $(7,202$ cemented and 7,202 cementless) enrolled in the National Joint Registry and the English National PROMs collection programme. Subgroup analyses were performed in different age groups (1) $<55$ years; (2) 55-64 years; (3) 65-74 years; (4) $\geq 75$ years.

Results - The 6-month postoperative Oxford Knee Score (OKS) was significantly $(\mathrm{p}<0.001)$ higher for cemented TKR (35, SD 9.7) than cementless TKR (34, SD 9.9). The OKS was also significantly higher for the cemented TKR in all age groups, except the 55-64-year group. A significantly higher proportion of cemented TKRs had an excellent OKS $(\geq 41)$ compared with cementless $(32 \%$ vs. $28 \%$, p < 0.001 ) and a lower proportion of poor $(<27)$ scores $(19 \%$ vs. $22 \%$, p $=0.001)$. This was also observed for all age subgroups. There were no significant differences in EQ-5D points gained postoperatively between the groups respectively $(0.31$ vs. 0.30 , $\mathrm{p}=0.1$.

Interpretation - Cemented TKRs had a greater proportion of excellent OKS scores and lower proportion of poor scores both overall and across all age groups. However, the absolute differences are small and below the minimally clinically important difference, making both fixation types acceptable. Currently the vast majority of TKRs are cemented and the results from this study suggest that this is appropriate.
Total knee replacement (TKR) can be implanted with (cemented) or without (cementless) bone cement. Although cemented fixation is the current gold standard, it has potential problems including cement embolisms, late implant loosening, and complex revision surgery, and cement polymerisation may contribute to chronic pain $(1,2)$.

Additionally, there is a change in the demographics of patients requiring joint replacements, to include more younger patients. The under 65 years group is predicted to be the main cohort by 2030 (3). These patients are generally more active and place greater functional demands on their knee replacements.

Traditionally the metric for measuring an implant's performance has been the revision rate. This metric allows straightforward comparison of implant types but does not give insight into functionality (4). In recent years there been a drive toward using more patient-relevant outcomes (5). Goodman et al. (6) found that the most important outcome measures to patients were the function, pain status, and quality of life following surgery. Currently 1 in 5 patients report clinically significant persistent pain and dissatisfaction following TKR (7).

There is no consensus on which TKR fixation method offers better functional outcomes with any research significantly limited by sample size. Although the use of cement in TKRs is well established, cementless fixation has several theoretical advantages including bone-stock preservation, reduced operative times, ease of revision, and elimination of the bone-cement interface. Indeed, RSA studies have suggested lower rates of aseptic loosening in cementless compared with cemented TKRs $(\mathbf{8 , 9})$.

We performed the largest study to date comparing the functional and quality of life outcomes of matched cemented 
and cementless TKRs using data from 3 national datasets: the National Joint Registry for England Wales and Northern Ireland (NJR); Hospital Episodes Statistics Admitted Patient Care records (HES-APC); and England's National Patient Reported Outcome Measures (PROMs) programme.

\section{Patients and methods}

\section{Data sources}

We performed a retrospective observational study using NJR records linked to the HES-APC database and PROMs database. The NJR was established in April 2003 and is the world's largest arthroplasty register (10). The NJR only has $1.5 \%$ missing data for primary knee replacements according to their data quality audits (10). HES-APC is a database of all admission episodes for patients being admitted to an NHS hospital in England (11). From approximately 2009 onwards, NHS-funded knee replacements as part of the PROMs programme have both preoperative and 6 months postoperative PROMs recorded (12). These include the Oxford Knee Score (OKS) (13) and quality of life index Euro Qol 5 Domain index (EQ-5D) $(14,15)$. The preoperative questionnaire is completed in person at the surgical unit and the 6-month questionnaire is posted to the patient independently of the operating unit.

The OKS has 12 items relating to knee pain and function, each scoring from 0 to 4 , presented as an overall score on an ordinal scale between 0 and 48 (13). The mean scores are given together with the proportion attaining excellent $(\geq 41$ points on the OKS), good (34-41), fair (27-33), and poor $(<27)$ results defined by Kalairajah et al. (16). The minimal clinically important difference (MCID) for the OKS has not been definitively quantified: it is thought to be between 3 and 5 points (13). The EQ-5D comprises 5 questions about mobility, self-care, activities of daily living, pain, and anxiety/ depression, each with three levels (no problems, some problems, severe problems). These answers can be presented as a weighted overall index rated from 1 (perfect health) to -0.594 (worst possible state) $(\mathbf{1 4 , 1 5})$.

The choice of time intervals in the PROMs programme was a compromise between proximity to surgery (to provide timely feedback and to avoid "contamination" with nonoperationrelated factors) and providing sufficient follow-up to allow comparison whilst accounting for the postoperative recovery period. The evidence indicates most improvement in PROMs after surgery occurs in the first 6 months, with only minor improvement between 6 months and 1 year (17). Long-term TKR studies have shown PROMs remain relatively constant after the early improvement, at least up to the 10th postoperative year $(18,19)$.

\section{Data linkage}

Between January 1, 2004 and December 31, 2018, 687,910 TKRs from the NJR dataset (10) were successfully linked to

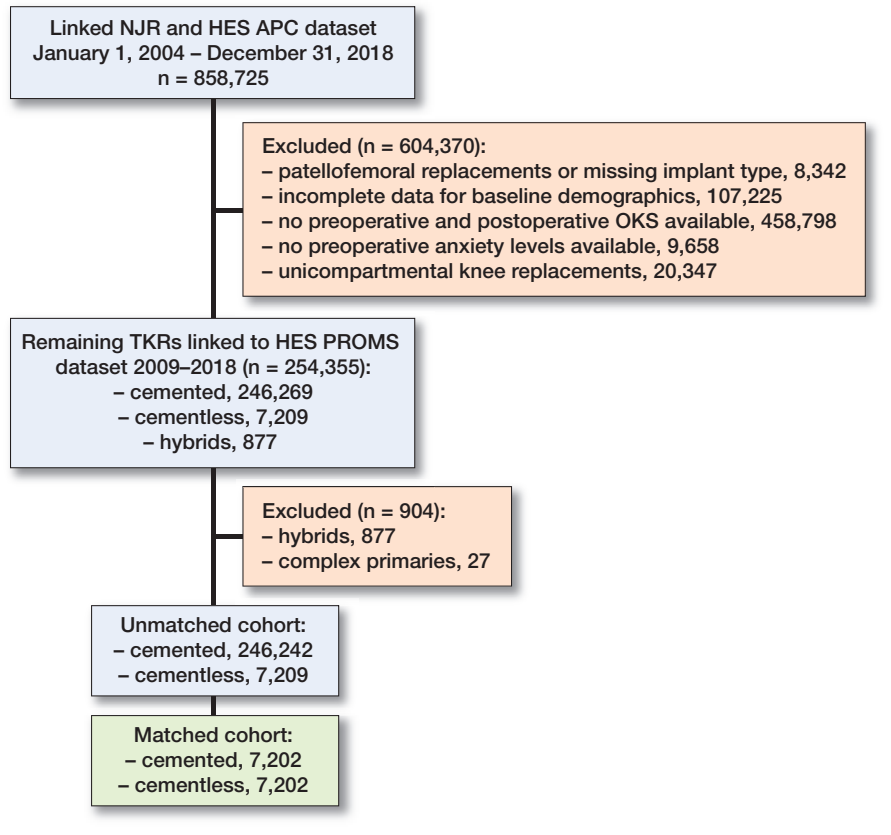

Figure 1. Dataset cleaning and merging.

the HES APC dataset (11) with a full set of baseline demographic and surgical factors needed for matching (Figure 1). This dataset was subsequently merged with the HES PROMs dataset, which started collecting data from approximately 2009 onwards (12). All preoperative PROMs needed to be completed within 3 months preoperatively from surgery or at the latest 1 month postoperatively to be regarded as robust for inclusion. Cases were excluded if either no preoperative anxiety score was available (given that this was needed for matching) or there was not both a preoperative and postoperative OKS score (Figure 1). There were 254,355 TKRs meeting the above criteria which was $37 \%$ of the NJR-HES-APC TKR cohort. This is to be expected given that the PROMs database only started collecting data from approximately 2009 onwards (12) whereas the NJR data in this study is from 2004. After removing hybrids and complex primary procedures there were 253,451 TKRs (246,242 cemented and 7,209 cementless) eligible for inclusion. All datasets were linked using pseudo-anonymised identification numbers.

\section{Statistics}

There were statistically significant differences in baseline characteristics between cemented and cementless TKR groups (Table 1). Given the potential for factors other than fixation to affect the outcomes we a priori matched the cemented and cementless groups for multiple known patient and surgical confounders using propensity scores. Logistic regression was used to generate a propensity score representing the probability that a patient received a cementless TKR and the data was generated from patient demographics and surgical factors (including surgeon caseload). All patient and surgical factors in Table 1 were used for matching, apart from BMI, which 
had a large proportion of missing data. This is a well-recognised approach for comparing implant types $(\mathbf{2 0 , 2 1 )}$. Surgical factors included surgeon caseload, defined as the average number of primary knee replacements performed per year. Every surgeon in the NJR has a specific identifier, which was used to calculate this as described previously (20). The matching algorithm matched on the logit of the propensity score with a $0.02-\mathrm{SD}$ calliper width at a 1:1 ratio. Greedy matching without replacement was used given that it has superior performance for estimating treatment effects (22). Standardized mean differences (SMDs) were examined both before and after matching to assess for any imbalance between groups, with SMDs of $10 \%$ or more considered suggestive of covariate imbalance (23). After matching, 14,404 knee replacements $(7,202$ cemented and 7,202 cementless TKRs) were available for analysis.

Outcomes of interest were: (1) preoperative OKS and EQ-5D scores, (2) 6-month postoperative OKS and EQ-5D scores, and (3) difference in OKS and EQ-5D scores postoperatively and preoperatively. Subgroup analyses were performed in 4 different age groups as per the NJR (10): $(1)<55$ years, (2) 55-64 years, (3) 65-74 years, and $(4) \geq 75$ years.

Given that the PROMs scores were not normally distributed, appropriate nonparametric tests were used to compare scores (Figure 2A and B). To compare pre- and postoperative scores within groups we used the Wilcoxon signed rank test. To compare fixation groups scores we used the Mann-Whitney test.

All statistical analyses were performed using Stata (Version 15.1; StataCorp, College Station, TX, USA) except propensity
Table 1. Baseline characteristics before and after matching cemented and cementless TKRs

\begin{tabular}{|c|c|c|c|c|c|c|}
\hline \multirow[b]{2}{*}{ Covariate } & \multicolumn{2}{|c|}{ Unmatched cohort } & \multicolumn{4}{|c|}{ Matched cohort } \\
\hline & $\begin{array}{c}\text { Cemented } \\
n=246,242\end{array}$ & $\begin{array}{c}\text { Cementless } \\
n=7,209\end{array}$ & SMD & $\begin{array}{l}\text { Cemented } \\
n=7,202\end{array}$ & $\begin{array}{c}\text { Cementless } \\
\mathrm{n}=7,202\end{array}$ & SMD \\
\hline \multicolumn{7}{|l|}{ Admission type } \\
\hline Elective & $246,072(100)$ & $7,204(100)$ & 0.01 & $7,197(100)$ & $7,197(100)$ & 0.02 \\
\hline Emergency & $159(0)$ & $4(0)$ & & $5(0)$ & $4(0)$ & \\
\hline Other & $11(0)$ & $1(0)$ & & $0(0)$ & $1(0)$ & \\
\hline \multicolumn{7}{|l|}{ Sex } \\
\hline Female & $140,879(57)$ & $3,642(51)$ & 0.14 & $3,648(51)$ & $3,640(51)$ & 0.002 \\
\hline Male & $105,363(43)$ & $3,567(49)$ & & $3,554(49)$ & $3,562(49)$ & \\
\hline \multicolumn{7}{|l|}{ Age at surgery } \\
\hline Mean (SD) & $70.2(8.8)$ & $69.3(8.9)$ & 0.11 & $69.2(9.0)$ & $69.3(8.9)$ & 0.01 \\
\hline BMI, mean (SD) & $30.9(5.4)$ & $30.7(5.0)$ & 0.05 & $30.8(5.2)$ & $30.7(5.0)$ & 0.03 \\
\hline $\mathrm{n}$ & 187,324 & 4,892 & & 5,343 & 4,887 & \\
\hline \multicolumn{7}{|l|}{ Primary diagnosis } \\
\hline Primary OA & $238,052(97)$ & $7,089(98)$ & 0.11 & $7,047(98)$ & $7,082(98)$ & 0.04 \\
\hline $\mathrm{OA}$ and other & $2,594(1)$ & $41(1)$ & & $53(1)$ & $41(1)$ & \\
\hline Other & $5,596(2)$ & $79(1)$ & & $102(1)$ & $79(1)$ & \\
\hline \multicolumn{7}{|c|}{ Charlson comorbidity index } \\
\hline None & $171,124(70)$ & $5,235(72)$ & 0.10 & $5,253(73)$ & $5,228(72)$ & 0.01 \\
\hline Mild & $52,318(21)$ & $1,499(21)$ & & $1,485(21)$ & $1,499(21)$ & \\
\hline Moderate & $15,783(6)$ & $335(5)$ & & $322(4)$ & $335(5)$ & \\
\hline Severe & $7,017(3)$ & $140(2)$ & & $142(2)$ & $140(2)$ & \\
\hline \multicolumn{7}{|l|}{ Ethnicity } \\
\hline White & $235,551(96)$ & $7,015(97)$ & 0.10 & $7,002(97)$ & $7,008(97)$ & 0.03 \\
\hline Black (Caribbean) & $1,265(0)$ & $21(0)$ & & $19(0)$ & $21(0)$ & \\
\hline Black (African) & $836(0)$ & $10(0)$ & & $9(0)$ & $10(0)$ & \\
\hline Black (Other) & $365(0)$ & $12(0)$ & & $10(0)$ & $12(0)$ & \\
\hline Indian & $4,402(2)$ & 79 (1) & & $81(1)$ & $79(1)$ & \\
\hline Pakistani & $1,256(0)$ & $20(0)$ & & $31(0)$ & $20(0)$ & \\
\hline Bangladeshi & $112(0)$ & $1(0)$ & & $3(0)$ & $1(0)$ & \\
\hline Chinese & $172(0)$ & $2(0)$ & & $2(0)$ & $2(0)$ & \\
\hline Other & $2,283(1)$ & $49(1)$ & & $45(1)$ & 49 (1) & \\
\hline \multicolumn{7}{|c|}{ Rural/urban classification } \\
\hline Urban & $181,445(74)$ & $5,467(76)$ & 0.09 & $5,432(75)$ & $5,462(76)$ & 0.01 \\
\hline Town/fringe & $30,810(12)$ & $690(10)$ & & $692(10)$ & $690(10)$ & \\
\hline Village/hamlet & 33,987 (14) & $1,052(14)$ & & $1,078(15)$ & $1,050(14)$ & \\
\hline \multicolumn{7}{|c|}{$\begin{array}{l}\text { Indices of multiple } \\
\text { deprivation (quintiles) }\end{array}$} \\
\hline 1 & 33,667 (14) & 849 (12) & 0.11 & 855 (12) & 849 (12) & 0.007 \\
\hline 2 & 44,021 (18) & $1,119(15)$ & & $1,119(16)$ & $1,118(15)$ & \\
\hline 3 & $54,962(22)$ & $1,564(22)$ & & $1,575(22)$ & $1,564(22)$ & \\
\hline 4 & $58,548(24)$ & $1,977(27)$ & & $1,974(27)$ & $1,972(27)$ & \\
\hline 5 & 55,044 (22) & $1,700(24)$ & & 1,679 (23) & $1,699(24)$ & \\
\hline \multicolumn{7}{|c|}{ Surgeon caseload of primary } \\
\hline knee surgery practi & & & & & & \\
\hline cases/years (SD) & $80.6(48.3)$ & $80.8(43.6)$ & 0.006 & $80.7(48.8)$ & $80.9(43.6)$ & 0.005 \\
\hline \multicolumn{7}{|l|}{ ASA grade } \\
\hline 1 & $21,270(9)$ & $892(12)$ & 0.17 & $968(13)$ & $887(12)$ & 0.03 \\
\hline 2 & $184,020(74)$ & $5,463(75)$ & & 5,407 (75) & $5,461(76)$ & \\
\hline 3 or above & $40,952(17)$ & $854(12)$ & & 827 (12) & $854(12)$ & \\
\hline \multicolumn{7}{|l|}{ VTE-chemical } \\
\hline LMWH ( \pm other) & $173,997(70)$ & $4,861(67)$ & 0.18 & $4,756(66)$ & 4,859 (67) & 0.04 \\
\hline Aspirin only & $12,106(5)$ & 203 (3) & & $236(3)$ & 203 (3) & \\
\hline Other & $53,581(22)$ & 2,019 (28) & & 2,069 (29) & $2,014(28)$ & \\
\hline None & $6,558(3)$ & $126(2)$ & & $141(2)$ & $126(2)$ & \\
\hline \multicolumn{7}{|l|}{ VTE-mechanical } \\
\hline Any & $234,699(95)$ & $6,901(96)$ & 0.02 & $6,907(96)$ & $6,895(96)$ & 0.008 \\
\hline None & 11,543 (5) & $308(4)$ & & 295 (4) & 307 (4) & \\
\hline
\end{tabular}

score matching, which was performed using R (Version 3.4.0; R Foundation for Statistical Computing, Vienna, Austria). 95\% confidence intervals (CI) are presented and statistical significance was defined at the $5 \%$ level. 
Table 1.Continued

\begin{tabular}{|c|c|c|c|c|c|c|}
\hline \multirow[b]{2}{*}{ Covariate } & \multicolumn{2}{|c|}{ Unmatched cohort } & \multicolumn{4}{|c|}{ Matched cohort } \\
\hline & $\begin{array}{c}\text { Cemented } \\
n=246,242\end{array}$ & $\begin{array}{c}\text { Cementless } \\
n=7,209\end{array}$ & SMD & $\begin{array}{l}\text { Cemented } \\
n=7,202\end{array}$ & $\begin{array}{c}\text { Cementless } \\
n=7,202\end{array}$ & SMD \\
\hline \multicolumn{7}{|l|}{ Year of surgery } \\
\hline 2008 & $6(0)$ & $0(0)$ & 0.34 & $0(0)$ & $0(0)$ & 0.14 \\
\hline 2009 & $12,996(5)$ & $804(11)$ & & $628(9)$ & $800(11)$ & \\
\hline 2010 & $21,257(8)$ & $951(13)$ & & $889(12)$ & 949 (13) & \\
\hline 2011 & 23,657 (10) & 909 (13) & & $960(13)$ & 909 (13) & \\
\hline 2012 & 24,117 (10) & 733 (10) & & 866 (12) & 732 (10) & \\
\hline 2013 & $26,471(11)$ & $622(9)$ & & 807 (11) & $622(9)$ & \\
\hline 2014 & $28,855(12)$ & $747(10)$ & & $808(11)$ & 747 (10) & \\
\hline 2015 & $28,351(11)$ & $754(10)$ & & $699(10)$ & $754(11)$ & \\
\hline 2016 & $29,222(12)$ & $625(9)$ & & $613(9)$ & $625(9)$ & \\
\hline 2017 & $27,051(11)$ & $615(9)$ & & $536(7)$ & 615 (8) & \\
\hline 2018 & 24,259 (10) & $449(6)$ & & $396(6)$ & $449(6)$ & \\
\hline \multicolumn{7}{|c|}{ Preoperative Oxford Knee } \\
\hline Score (SD) & $18.9(7.7)$ & $19.2(7.8)$ & 0.04 & $19.2(7.6)$ & $19.2(7.8)$ & 0.005 \\
\hline \multicolumn{7}{|c|}{$\begin{array}{l}\text { Preoperatively anxious } \\
\text { or depressed }\end{array}$} \\
\hline No & $155,409(63)$ & $4,629(64)$ & 0.02 & $4,631(64)$ & $4,623(64)$ & 0.003 \\
\hline Moderately & 81,336 (33) & 2,321 (32) & & 2,317 (32) & 2,321 (32) & \\
\hline Extremely & $9,497(4)$ & $259(4)$ & & $254(4)$ & $258(4)$ & \\
\hline \multicolumn{7}{|c|}{ Bone graft used } \\
\hline No & $243,155(99)$ & $7,045(98)$ & 0.08 & $7,029(98)$ & $7,044(98)$ & 0.01 \\
\hline Yes & $3,087(1)$ & $164(2)$ & & $173(2)$ & $158(2)$ & \\
\hline
\end{tabular}

Ethics, funding, and potential conflicts of interest

This study was approved by the NJR Research subcommittee and had ethical approval from the South Central Oxford B Research Ethics Committee (19/SC/0292). The linkage of the datasets was approved by the Confidentiality Advisory Group (19/CAG/0054). Financial support has been received from Zimmer Biomet. HRM was supported by the Henni Mester Scholarship at University College, Oxford University and the Royal College of Surgeons' Research Fellowship. AJ was supported by the NIHR Biomedical Research Centre at the University Hospitals Bristol NHS Foundation Trust and the University of Bristol.
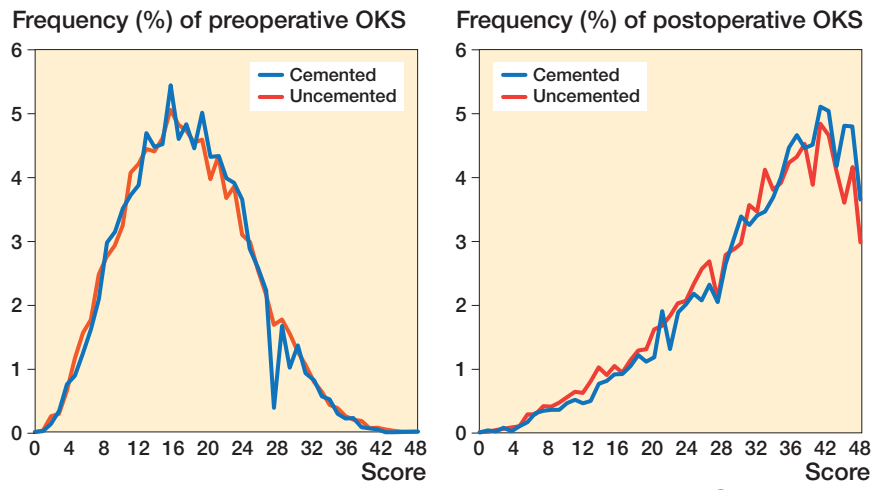

Figure 2. Distribution of preoperative and postoperative Oxford Knee Scores.

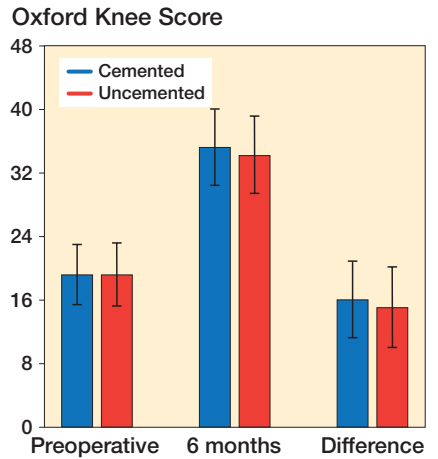

Figure 3. Comparison of OKS in matched cohort of cemented and cementless TKRs.

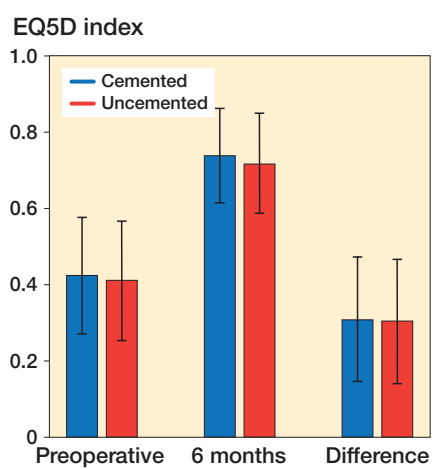

Figure 4. Comparison of EQ-5D index in matched cohort of cemented and cementless TKRs.

\section{Results}

The unmatched cohort consisted of 246,242 cemented and 7,209 cementless TKRs. There were statistically significant baseline differences in the patient and surgical factors between groups (Table 1). The matched study group consisted off 14,404 knee replacements $(7,202$ cemented and 7,202 cementless TKRs) with both groups well balanced for patient and surgical factors (Table 1).

The mean preoperative OKSs for the matched cemented and cementless groups were 19 (SD 7.6) and 19 (SD 7.8) $(\mathrm{p}=0.5)$. Both groups showed improvements in their 6-month postoperative scores ( $p<0.001)$ to 35 (SD 9.7) and 34 (SD 9.9) respectively. The cemented group had a higher $(\mathrm{p}<0.001) 6$-month postoperative score by 1 point. The cemented group gained 16 points (SD 9.8) postoperatively whereas the cementless group gained 15 points (SD 10.1) $(\mathrm{p}<0.001)$ (Figure 3$)$. The cemented group had a higher proportion of postoperative excellent OKS compared with the cementless group (32\% vs. $28 \%, \mathrm{p}<0.001)$ and lower proportion of poor postoperative OKS (19\% vs. $22 \%, \mathrm{p}=0.001)$ (Table 2$)$.

The mean preoperative EQ-5D index scores for the cemented and cementless groups were 0.42 (SD 0.31) and 0.41 (SD 0.31) $(\mathrm{p}=0.1)$. Both groups showed improvements in their postoperative scores $(\mathrm{p}<0.001)$ to 0.74 (SD 0.25$)$ and 0.72 (SD 0.26). The cemented group gained 0.31 (SD 0.32) points postoperatively and the cementless group gained 0.30 $(\mathrm{SD} 0.33)(\mathrm{p}=0.1)($ Figure 4$)$. 
Table 2. Proportion of Oxford Knee Scores as per the Kallairajah et al. (16) classification in the matched cemented and cementless TKR groups. Values are count (\%)

\begin{tabular}{lccc}
\hline $\begin{array}{l}\text { Oxford Knee Score } \\
\text { categorisation }\end{array}$ & $\begin{array}{c}\text { Cemented } \\
(\mathrm{n}=7,202)\end{array}$ & $\begin{array}{c}\text { Cementless } \\
(\mathrm{n}=7,202)\end{array}$ & p-value \\
\hline $\begin{array}{l}\text { Preoperatively } \\
\text { Poor }\end{array}$ & $5,955(83)$ & $5,891(82)$ & 0.7 \\
$\quad$ Fair & $974(13)$ & $1,006(14)$ & 0.5 \\
$\quad$ Good & $264(4)$ & $286(4)$ & 0.4 \\
$\quad$ Excellent & $9(0)$ & $19(0)$ & 0.06 \\
Postoperatively & & & \\
$\quad$ Poor & $1,351(19)$ & $1,547(22)$ & 0.001 \\
Fair & $1,273(18)$ & $1,319(18)$ & 0.4 \\
Good & $2,265(31)$ & $2,303(32)$ & 0.6 \\
Excellent & $2,313(32)$ & $2,033(28)$ & $<0.001$ \\
\hline
\end{tabular}

Table 3. Pre- and post-operative OKS (SD) in different age groups for matched cemented and cementless TKR groups

\begin{tabular}{lccccccc}
\hline \multirow{2}{*}{$\begin{array}{l}\text { Age } \\
\text { group }\end{array}$} & \multicolumn{2}{c}{ Cemented, score } & \multicolumn{5}{c}{ Cementless, score } \\
preop. & 6-month & $\Delta$ & preop. & 6-month & $\Delta$ & Diff \\
\hline$<55$ & $18(7.5)$ & $33(11)$ & 15 & $16(7.2)$ & $30(11)$ & 14 & 3 \\
$55-64$ & $18(7.2)$ & $34(11)$ & 16 & $18(7.5)$ & $33(10)$ & 15 & 1 \\
$65-74$ & $20(7.6)$ & $36(9.2)$ & 16 & $20(7.8)$ & $35(9.7)$ & 15 & 1 \\
$\geq 75$ & $20(7.7)$ & $36(8.9)$ & 16 & $20(8.0)$ & $35(9.3)$ & 15 & 1 \\
\hline
\end{tabular}

$\Delta=6$-month score - preoperative score

Diff $=$ Difference between 6-month cemented and cementless score

\section{Effect of age on PROMS in the matched cohort}

Age groups were stratified into 4 groups as per the NJR report: (1) < 55 years (421 cemented, 435 cementless); (2) 55-64 years (1,704 cemented, 1,655 cementless); (3) 65-74 years (2,980 cemented, 2,990 cementless); and (4) $\geq 75$ years $(2,097$ cemented, 2,122 cementless).

Preoperatively the mean OKS was similar between fixation groups across all age groups except the $<55$ years, where the cementless group has a slightly lower score. For both fixation groups, younger groups had poorer OKS than older groups
(Table 3). All age groups showed improvements postoperatively compared with preoperatively in both fixation groups ( $\mathrm{p}$ $<0.001$ ) (Table 3). The 6-month OKS was higher in cemented compared with cementless TKRs in age groups $<55$ years ( $\mathrm{p}$ $<0.001), 65-74$ years $(\mathrm{p}<0.001)$, and $\geq 75$ years $(\mathrm{p}=0.01)$. Scores for the group 55-64 years were similar $(\mathrm{p}=0.1)$ (Table 3 ). Cemented TKRs gained more points postoperatively compared with cementless TKRs in age groups 65-74 years $(\mathrm{p}<$ $0.001)$ and $\geq 75$ years $(p=0.003)$. In the other age groups, the points gained were similar (Table 3 ).

Preoperatively the proportions of poor, fair, good, and excellent OKS were similar between fixation groups for all age groups except the $<55$ years where there was a greater proportion of poor scores in the cementless group (Table 4). The proportion of 6-month postoperative excellent scores was $10 \%, 3 \%, 5 \%$, and $2 \%$ higher in cemented compared with cementless group for the $<55$ years, 55-64 years, 65-74 years, and $\geq 75$ years groups respectively. The proportion of 6-month postoperative poor OKS scores was $4 \%, 0 \%, 4 \%$, and $4 \%$ lower in cemented compared with cementless TKRs across the respective age groups.

Preoperatively the EQ-5D score was similar between fixation groups across all ages except in the $<55$ years group where it was higher for the cemented group (Table 5). For both cemented and cementless TKRs younger age groups had poorer EQ-5D scores than older groups. For both cemented and cementless TKRs all age groups showed statistically significant improvements postoperatively compared with preoperatively $(\mathrm{p}<0.001)$. The points gained between the cemented and cementless groups were similar across all age groups (Table 5). The 6-month EQ-5D was higher in all age groups for cemented compared with cementless TKRs (Table 5).

\section{Discussion}

This is the largest study to date comparing the PROMs of cemented and cementless TKRs. The most important finding was that, after matching, the cemented TKR had statistically significantly higher mean OKS, although the absolute difference was small and below the MCID for the OKS. However, a greater proportion of excellent OKS $(32 \%$ vs. $28 \%)$ and lower proportion of poor OKS (19\% vs. $22 \%)$ were achieved by cemented fixation.

The postoperative OKS of cemented implants was 
Table 5. Pre- and postoperative EQ5D index scores (SD) in different age groups for cemented and cementless TKR groups

\begin{tabular}{lccccccc}
\hline \multirow{2}{*}{$\begin{array}{l}\text { Age } \\
\text { group }\end{array}$} & \multicolumn{2}{c}{ Cemented, score } & preop. & 6-month & $\Delta$ & \multicolumn{2}{c}{ Cementless, score } \\
preop. & 6-month & $\Delta$ & Diff \\
\hline$<55$ & $0.36(0.32)$ & $0.66(0.32)$ & 0.30 & $0.29(0.32)$ & $0.58(0.32)$ & 0.30 & 0.08 \\
$55-64$ & $0.39(0.31)$ & $0.69(0.28)$ & 0.31 & $0.38(0.32)$ & $0.68(0.27)$ & 0.30 & 0.01 \\
$65-74$ & $0.45(0.30)$ & $0.76(0.23)$ & 0.32 & $0.43(0.31)$ & $0.74(0.25)$ & 0.31 & 0.02 \\
$\geq 75$ & $0.44(0.30)$ & $0.75(0.22)$ & 0.32 & $0.44(0.31)$ & $0.74(0.23)$ & 0.30 & 0.01 \\
\hline
\end{tabular}

$\Delta=6$-month score - preoperative score

Diff $=$ Difference between 6-month cemented and cementless score

statistically significantly higher than cementless by 1 point and this difference is below the theoretical MCID, which lies between 3 to 5 points (24). However, the skewed nature and ceiling effect of the OKS (25) limits the usefulness of comparing postoperative mean scores. Thus the difference may be clinically important. It is not clear why there is a difference. It may be a manifestation of the more reliable fixation achieved by cement in the early phase postoperatively. Nam et al. (26) performed an RCT comparing cemented and cementless TKRs and found no statistically significant difference in OKS at 6 weeks, 1 year, and 2 years postoperatively. Prasad et al. (27) more recently performed a metaanalysis comparing the midterm outcomes of cemented and cementless TKRs and also found no differences in the Western Ontario and McMaster Universities Osteoarthritis Index or Knee Society Score between groups. Additionally, Rassir et al. (28) in a multicentre study found that the cemented and cementless TKRs did not differ statistically significantly in functional outcomes.

There is some concern regarding cemented fixation in younger patients given that they have longer life expectancies and higher postoperative activities. Cement cannot remodel like a biological integrated component, which can potentially lead to higher rates of loosening resulting in pain and functional impairment, particularly in younger patients. This has renewed interest in cementless that employ new technologies such as hydroxyapatite and trabecular metal (27). A Cochrane review has shown aseptic loosening rates to be statistically significantly lower in cementless TKR compared with cemented (8). Although cementless TKR implants do migrate there is evidence that this stabilises at 3 months whereas cemented TKRs continue to migrate (9). In our study, the cemented group performed statistically significantly better for both the OKS and the EQ-5D index in the < 55 years group. Furthermore, the advantage of cemented over cementless tended to be larger in the $<55$ years than the older groups. This is important because it tends to contradict the generally held view that cementless fixation is most appropriate for young patients.

Our study showed the mean 6-month EQ-5D scores for cemented and cementless TKR was 0.74 and 0.72 respectively. The 6-month EQ-5D was statistically significantly higher in all age groups for cemented TKRs except in the over-75 group. This is in contrast to work by Rassir et al. (28) who found no statistically significant difference in the EQ-5D or cost-effectiveness between cemented and cementless TKRs in a small study. The absolute difference in EQ-5D of 0.02 observed in our study is small, and is lower than 0.03 which is the lower end of the MCID for the EQ-5D index described by Coretti et al. (29) for musculoskeletal related issues. However, when cumulated over the many years that knee replacements are expected to function this would increase the number QALY (quality adjusted life years) achieved by cemented TKR compared with cementless TKR. Taken together with lower 10-year revision rate of cemented TKRs (3.4\% vs. $4.2 \%$ ) according to the NJR (10) and the lower cost of cemented components, cemented TKRs are likely to be more cost-effective.

The strengths of this study are that it is an unselected registry sample recruited over an extended 10-year period, which reduces the chances of selection bias. By linking the NJR, HES-APC, and PROMS dataset, various patient and surgical confounding factors were matched to allow a fair comparison of similar population cohorts. The main limitation is this study is retrospective and based on observational data. We did not match cemented and cementless TKRs on implant type and therefore there may be unmeasured confounders that could influence our results. Matching can reduce the generalisability of findings, but as we were able to match virtually all the cementless to cemented TKRs this is unlikely to be an issue. The only way to achieve balance with respect to both known and unknown confounders is with a randomised trial. A limitation of our study is that only $37 \%$ of the TKRs from the NJRHES APC dataset had linked PROMs data available for analysis. However, this is to be expected given that the PROMs database only started collecting data from approximately 2009 onwards (12) whereas the NJR data in this study is from 2004. Additionally, TKRs vary in their design and material, and in our study they have all been pooled. This should not, however, influence our results as we have included all TKRs implanted over the study period. A further limitation is that the postoperative scores were collected at 6 months, though research suggests that any difference found at 6 months will persist in the long term (17-19).

In conclusion, cemented TKRs had a greater proportion of excellent OKS scores and lower proportion of poor scores both overall and across all age groups. However, the absolute differences in mean PROMs between cemented and cementless TKRs were small and below the MCID, making both fixation types acceptable. Currently over $95 \%$ of TKRs are cemented (10) and the results from this study and the lower cemented TKR revision rates reported in the NJR (10) suggest that this is appropriate. 
HRM, AJ, and DWM designed the study. HRM and DWM analysed the data with statistical support from AJ. HRM, AJ, and DWM helped with data interpretation. HRM wrote the initial manuscript draft, which was then revised appropriately by all authors.

The authors would like to thank the patients and staff of all the hospitals in England, Wales, Northern Ireland, and Isle of Man who have contributed data to the National Joint Registry. They are grateful to the Healthcare Quality Improvement Partnership (HQIP), the NJR Research Sub-committee, and staff at the NJR Centre for facilitating this work. The authors have conformed to the NJR's standard protocol for data access and publication. The views expressed represent those of the authors and do not necessarily reflect those of the National Joint Registry Steering Committee or the Healthcare Quality Improvement Partnership (HQIP), who do not vouch for how the information is presented.

Acta thanks Kirill Gromov and Gary Hooper for help with peer review of this study.

1. Kwong L, Nielsen E, Ruiz D, Hsu A, Dines M, Mellano C. Cementless total knee replacement fixation: a contemporary durable solution - affirms. Bone Joint J 2014; 96: 87-92. doi: 10.1302/0301-620X.96B11.34327.

2. McMahon S, Hawdon G, Bare J, Sim Y, Bertollo N, Walsh W. Thermal necrosis and PMMA: a cause for concern? Orthopaedic Proceedings 2012: 94-B(Suppl. XXIII): 64.

3. Kurtz S M, Lau E, Ong K, Zhao K, Kelly M, Bozic K J. Future young patient demand for primary and revision joint replacement: national projections from 2010 to 2030. Clin Orthop Relat Res 2009; 467: 2606-12. doi: 10.1007/s11999-009-0834-6.

4. Goodfellow $\mathbf{J}, \mathbf{O}^{\prime} \mathbf{C}$ Connor $\mathbf{J}$, Murray D. A critique of revision rate as an outcome measure: re-interpretation of knee joint registry data. Bone Joint J 2010; 92: 1628-31. doi: 10.1302/0301-620X.92B12.25193.

5. James Lind Alliance. Hip \& knee replacement for osteoarthritis top 10. 2017. Available online from: http://www.jla.nihr.ac.uk/priority-settingpartnerships/hip-and-knee-replacement-for-osteoarthritis/top-10-priorities/ (Accessed March 1, 2020).

6. Goodman S M, Mehta B, Mirza S Z, Figgie M P, Alexiades M, Rodriguez J, et al. Patients' perspectives of outcomes after total knee and total hip arthroplasty: a nominal group study. BMC Rheumatol 2020; 4: 3. doi: 10.1186/s41927-019-0101-8

7. Beswick A D, Wylde V, Gooberman-Hill R, Blom A, Dieppe P. What proportion of patients report long-term pain after total hip or knee replacement for osteoarthritis? A systematic review of prospective studies in unselected patients. BMJ Open 2012; 2 (1): e000435. doi: 10.1136/ bmjopen-2011-000435

8. Nakama G Y, Peccin M S, Almeida G J, Neto O de AL, Queiroz A A, Navarro R D. Cemented, cementless or hybrid fixation options in total knee arthroplasty for osteoarthritis and other non-traumatic diseases. Cochrane Database Syst Rev 2012; Oct 17; 10:CD006193. doi: 10.1002/14651858.CD006193.pub2.

9. Henricson A, Linder L, Nilsson K G. A trabecular metal tibial component in total knee replacement in patients younger than 60 years: a two-year radiostereophotogrammetric analysis. Bone Joint J 2008; 90: 1585-93. doi: 10.1302/0301-620X.90B12.20797.

10. National Joint Registry. National Joint Registry 18th annual report. National Joint Registry for England, Wales, Northern Ireland and Isle of Man; 2021.

11. NHS Digital. Hospital admitted patient care activity 2019-20. NHS Digital. Available online from: https://digital.nhs.uk/data-and-information/ publications/statistical/hospital-admitted-patient-care-activity/2019-20 (Accessed October 4, 2020).

12. NHS Digital. Patient reported outcome measures (PROMs). NHS Digital; 2020. Available online from: https://digital.nhs.uk/data-and-information/data-tools-and-services/data-services/patient-reported-outcomemeasures-proms (Accessed October 4, 2020).
13. Murray D, Fitzpatrick R, Rogers K, Pandit H, Beard D, Carr A, et al. The use of the Oxford hip and knee scores. Bone Joint J 2007; 89: 101014. doi: 10.1302/0301-620X.89B8.19424.

14. Group T E.EuroQol: a new facility for the measurement of health-related quality of life. Health Policy 1990; 16: 199-208. doi: 10.1016/01688510(90)90421-9.

15. Devlin N J, Parkin D, Browne J. Patient-reported outcome measures in the NHS: new methods for analysing and reporting EQ-5D data. Health Econ 2010; 19: 886-905. doi: 10.1002/hec.1608.

16. Kalairajah Y, Azurza K, Hulme C, Molloy S, Drabu K J. Health outcome measures in the evaluation of total hip arthroplasties: a comparison between the Harris hip score and the Oxford hip score. J Arthroplasty 2005; 20: 1037-41. doi: 10.1016/j.arth.2005.04.017.

17. Browne J P, Bastaki H, Dawson J. What is the optimal time point to assess patient-reported recovery after hip and knee replacement? A systematic review and analysis of routinely reported outcome data from the English patient-reported outcome measures programme. Health Qual Life Outcomes 2013; 11: 128. doi: 10.1186/1477-7525-11-128.

18. Williams D, Blakey C, Hadfield S, Murray D, Price A, Field R. Longterm trends in the Oxford knee score following total knee replacement. Bone Joint . 2013; 95: 45-51. doi: 10.1302/0301-620X.95B1.28573.

19. Breeman S, Campbell M, Dakin H, Fiddian N, Fitzpatrick R, Grant A, et al. Five-year results of a randomised controlled trial comparing mobile and fixed bearings in total knee replacement. Bone Joint J 2013; 95: 486-92. doi: 10.1302/0301-620X.95B4.29454.

20. Mohammad H R, Matharu G S, Judge A, Murray D W. The effect of surgeon caseload on the relative revision rate of cemented and cementless unicompartmental knee replacements: an analysis from the National Joint Registry for England, Wales, Northern Ireland and the Isle of Man. J Bone Joint Surg Am 2020; 102(8): 644-53. doi: 10.2106/ JBJS.19.01060.

21. Matharu G S, Judge A, Murray D W, Pandit H G. Outcomes after metal-on-metal hip revision surgery depend on the reason for failure: a propensity score-matched study. Clin Orthop Relat Res 2018; 476: 24558. doi: 10.1007/s11999.0000000000000029.

22. Austin P C. Some methods of propensity-score matching had superior performance to others: results of an empirical investigation and Monte Carlo simulations. Biom J 2009; 51: 171-84. doi: 10.1002/bimj. 200810488.

23. Austin P C. Balance diagnostics for comparing the distribution of baseline covariates between treatment groups in propensity-score matched samples. Stat Med 2009; 28: 3083-107. doi: 10.1002/sim.3697.

24. Beard D J, Harris K, Dawson J, Doll H, Murray D W, Carr A J, et al. Meaningful changes for the Oxford hip and knee scores after joint replacement surgery. J Clin Epidemiol 2015; 68: 73-9. doi: 10.1016/j. jclinepi.2014.08.009.

25. Dawson J, Beard D J, McKibbin H, Harris K, Jenkinson C, Price A. Development of a patient-reported outcome measure of activity and participation (the OKS-APQ) to supplement the Oxford knee score. Bone Joint J 2014; 96: 332-8. doi: 10.1302/0301-620X.96B3.32845.

26. Nam D, Lawrie C M, Salih R, Nahhas C R, Barrack R L, Nunley R M. Cemented versus cementless total knee arthroplasty of the same modern design: a prospective, randomized trial. J Bone Joint Surg Am 2019; 101: 1185. doi: 10.2106/JBJS.18.01162.

27. Prasad A K, Tan J H, Hanna S A, Dawson-Bowling S, Bedair H S. Cemented vs. cementless fixation in primary total knee arthroplasty: a systematic review and meta-analysis. EFORT Open Rev 2020; 5: 793-8. doi: 10.1302/2058-5241.5.200030

28. Rassir R, Nolte P, van der Lugt J, Nelissen R, Sierevelt I, Verra W. No differences in cost-effectiveness and short-term functional outcomes between cemented and uncemented total knee arthroplasty. BMC Musculoskelet Disord 2020; 21: 1-7. doi: 10.1186/s12891-020-03477-x.

29. Coretti S, Ruggeri M, McNamee P. The minimum clinically important difference for EQ-5D index: a critical review. Expert Rev Pharmacoecon Outcomes Res 2014; 14: 221-33. doi: 10.1586/14737167.2014. 894462. 